\title{
ELECTRO-OPTIC LONGITUDINAL ELECTRON BEAM DIAGNOSTIC AT SDL
}

\author{
H. Loos*, A. Doyuran, J. B. Murphy, J. Rose, T. Shaftan, B. Sheehy, \\ Y. Shen, J. Skaritka, X.J. Wang, Z. Wu, L.H. Yu, \\ BNL, Upton, NY 11973, USA
}

\begin{abstract}
The linac at the NSLS Source Development Lab (SDL) provides a high brightness electron beam for the DUV-FEL project with subpicosecond bunch length and several hundred Amperes peak current by means of a photoinjector and a magnetic bunch compressor. Previous diagnostics of the longitudinal bunch dynamics relied on the rf zero-phasing method and measurements of CTR spectra. In order to have a fast and non-intercepting longitudinal diagnostic available, the electro-optic measurement technique has been implemented with its major component, a synchronized $100 \mathrm{fs}$ Ti:Sapphire laser coaligned with the electron beam, already in place as a seed for the FEL. The theoretical temporal resolution for a 100 um thick ZnTe crystal is limited to about $200 \mathrm{fs}$ and the signal contrast to more than $1 \%$. We present preliminary results of multi-shot scanning measurements and the single-shot diagnostics of the bunch shape as well as its application as a rf-laser jitter monitor.
\end{abstract}

\section{INTRODUCTION}

The DUV-FEL accelerator [1] consists of a photoinjector, four SLAC-type accelerating structures for up to $200 \mathrm{MeV}$ beam energy, a magnetic chicane after the second linac tank compressing the beam to several hundred femtoseconds, and two undulators, a short modulator and NISUS, for self amplified spontaneous emission (SASE) and high gain harmonic generation (HGHG) experiments. The frequency tripled Ti:Sapphire laser for the photo-cathode generates several hundred $\mathrm{pC}$ of charge at a repetition rate of up to $10 \mathrm{~Hz}$. A small fraction of the fundamental serves as a seed for the HGHG experiment. The bunch length of the electron beam is regularly determined using a zero-phasing method [2], i.e. accelerating the beam with the last linac tank $90^{\circ}$ off crest and measuring the energy spectrum with a magnet spectrometer. However, the observed spikes in the temporal distribution cannot unambiguously be attributed to the time or energy domain with this method. Therefore, as a method based fully in the time domain, an electro-optic detection scheme $[3,4]$ has been installed, which is nondestructive and provides single-shot measurements to obtain the bunch shape. The induced birefringence in a ZnTe crystal from the Coulomb field of the electron beam is probed with the seed laser, which is synchronized with the electron beam, thus making measure-

*1oos@bnl.gov ments of both the temporal profile and the timing jitter between electron beam and seed laser available.

\section{ELECTRO-OPTIC DIAGNOSTIC}

The experimental set-up is located between the first spectrometer dipole after the accelerator and the modulator magnet of the HGHG experiment, where a temporal and transverse overlap of the electron beam and the seed laser can be established. As shown in Fig. 2, the electron beam bypasses the in-vacuum mirror for the seed laser by using four trim coils. A motor-controlled mount holds both the ZnTe crystal with the out-coupling mirror behind as well as a YAG screen (not shown). Depending on the mount's position, either the ZnTe crystal intercepts the laser with the electron beam passing by, or both the laser and the electron beam can be observed with a CCD camera imaging the YAG crystal. The seed laser and the Coulomb field of the electron beam at the ZnTe-crystal's location are horizontally polarized. For a $\langle 110\rangle$-cut crystal with the [001]-axis oriented vertically, the induced phase change is

$$
\Delta \varphi=\frac{2 \pi n_{0}^{3} r_{41} E_{\mathrm{vac}} l}{(1+\sqrt{\epsilon}) \lambda}
$$

with crystal length $l=500 \mu \mathrm{m}$, wavelength $\lambda=800 \mathrm{~nm}$, refractive index $n_{0}=2.853$, dielectric constant $\epsilon=$ 10.1 , and electro-optic coefficient $r_{41}=4.04 \mathrm{pm} / \mathrm{V}$. The Coulomb field at distance $r$ is

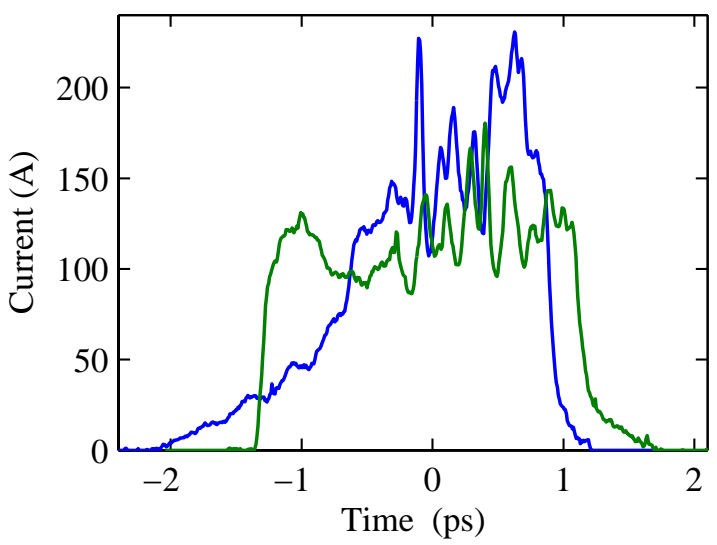

Figure 1: Time profile of the compressed electron beam using zero-phasing method. The two profiles are taken with $+90^{\circ}$ and $-90^{\circ}$ phase in the last linac tank. 


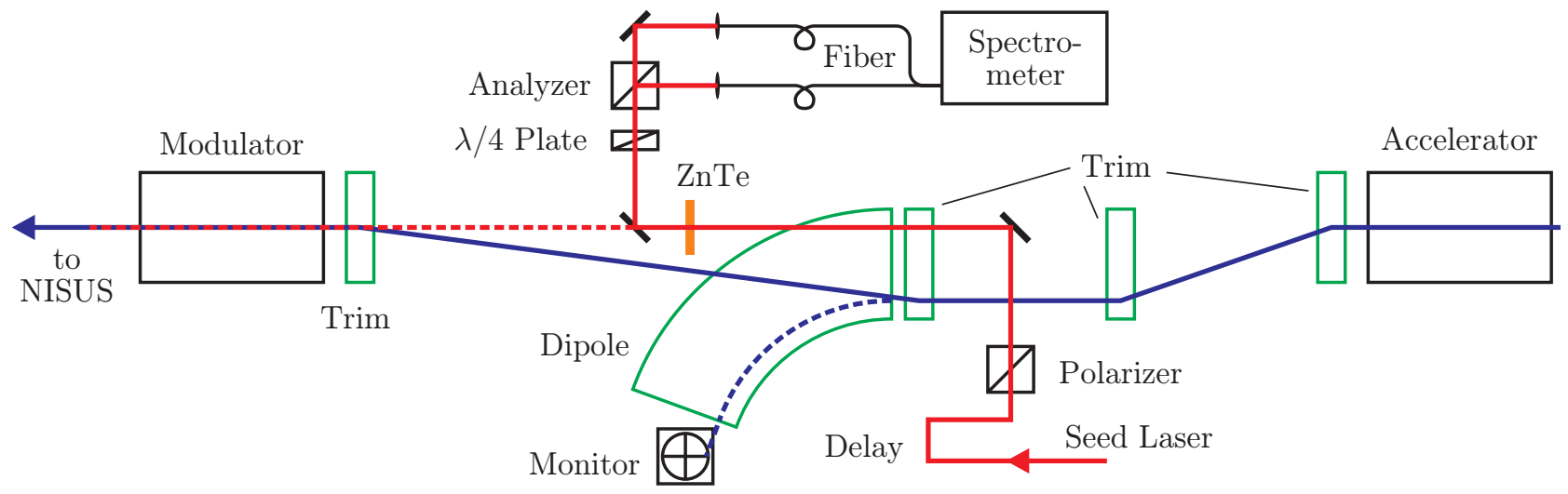

Figure 2: Setup of the electro-optical bunch length measurement placed in the section of the DUV-FEL accelerator between the last accelerating tank on the right and the HGHG modulator on the left.

$$
E_{\mathrm{vac}}=\frac{Z_{0} I}{2 \pi r}
$$

with the time dependent beam current $I$. The phase change is detected with a $\lambda / 4$ plate as compensator and an analyzer cube, whose transmitted and reflected beams are either focused into a pair of photodiodes for the multi-shot experiment or into a pair of fibers and eventually a monochromator with CCD array for the single-shot set-up [5]. With the compensator set to balanced intensities, the asymmetry of the integrated photodiode signals or the spectra becomes

$$
A=\frac{S_{\mathrm{T}}-S_{\mathrm{R}}}{S_{\mathrm{T}}+S_{\mathrm{R}}}=\sin \left(\Delta \varphi+\varphi_{0}\right),
$$

and $\varphi_{0}$ accounting for any residual birefringence.

\section{SCANNING EXPERIMENT}

The accelerator and seed laser were operated at their usual settings for the HGHG experiment with $177 \mathrm{MeV}$ beam energy, a bunch charge of $290 \mathrm{pC}$, and the seed laser chirped to $5.9 \mathrm{ps}$ (FWHM) for good overlap with the $1 \mathrm{ps}$ long electron bunches. The amplified difference signal of

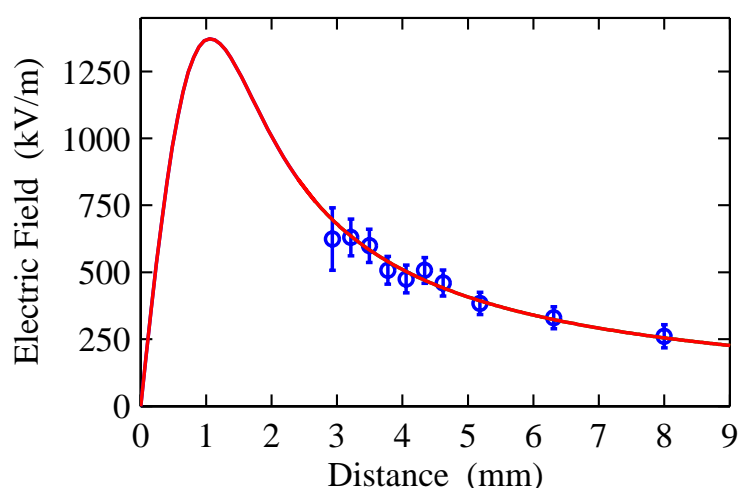

Figure 3: Electric field at maximum temporal overlap for different laser-electron beam distance with theoretical calculation as solid line. the photodiodes was averaged over 50 bunches for each data point, whereas the sum signal was measured only once. To eliminate electrical noise and background from electrons hitting the crystal, data was also taken with either the seed laser or the electron beam blocked. Figure 3 shows the effective electric field calculated from the signal asymmetry using Eq. (1) for different separations of laser and electron beam. Note that the obtained Coulomb field is reduced, since it is averaged over the much longer laser pulses. The solid curve shows Eq. (2) convoluted with the measured rms beam sizes of $300 \mu \mathrm{m}$ and $600 \mu \mathrm{m}$ for electron beam and laser, respectively. To fit the data points, the charge was assumed to be $230 \mathrm{pC}$, which is not far less than the $290 \mathrm{pC}$ measured in the Faraday cup.

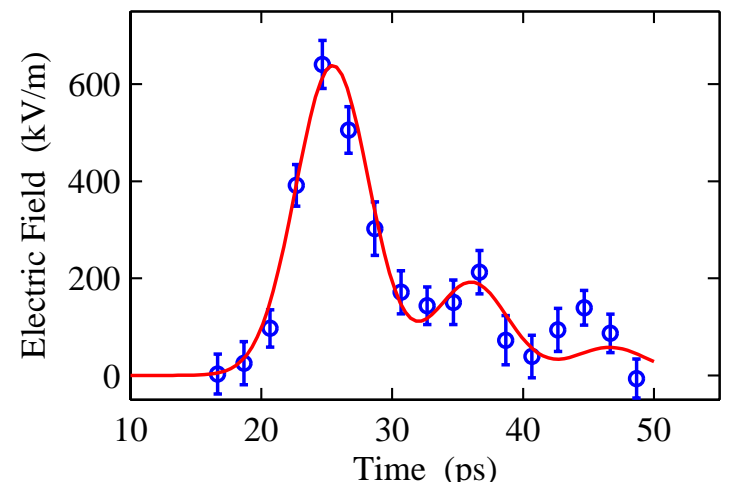

Figure 4: Electric field for different laser-electron beam delay with solid line as theoretical model.

A scan of the seed laser delay relative to the electron beam is shown in Figure 4. The main peak has a FWHM of $6.7 \mathrm{ps}$ which is slightly longer than the laser pulse. The solid curve is a corresponding gaussian distribution with satellites after multiples of $10 \mathrm{ps}$ added, which is the roundtrip time of THz waves in the ZnTe crystal. The peak height of the satellites is larger than expected which indicates the presence of trailing wake fields. No electric field ahead of the bunch could be observed. 


\section{SINGLE-SHOT EXPERIMENT}

The raw spectra, with and without electron beam, from the two multi-mode fibers carrying the transmitted and reflected beams from the analyzer cube, are shown in Fig. 5. Both reference spectra differ significantly from each other and their difference varies with the compensator orientation. This indicates a large wavelength dependent intrinsic birefringence, which partly is stress induced in the ZnTe crystal and partly may originate from a crystalline quartz vacuum window. The static phase is determined from the asymmetry of the reference spectra without electron beam and subtracted from the phase including the electro-optic signal. The resulting current profile using Eqs. $(1,2)$ is shown in Fig. 6 with a delay difference of 1.67,ps between the upper and lower part. The wavelength shift in the signal peak was used to obtain the time calibration to $\eta=(1.25 \pm 0.2) \mathrm{nm} / \mathrm{ps}$ which is close to the chirp in the seed laser of $1.15 \mathrm{~nm} / \mathrm{ps}$. The data in the lower part of the figure exhibits the expected single-sided shape of the Coulomb field, whereas each of the data in the upper part contains an additional pre- and post-pulse, which is not yet understood. Therefore, the following discussion only refers to the first set of data.

Considering the temporal resolution of the electro-optic set-up, the rms pulse width of this measurement of (730 \pm $75)$ fs can be compared with the bunch length of (625 \pm 50) fs obtained with the zero-phasing method, which has a resolution of tens of femtoseconds. The individual FWHM contributions for the the electro-optic method are the intrinsic duration of the Coulomb field in $2.1 \mathrm{~mm}$ distance of $\tau_{r}=r /(\gamma c)=20 \mathrm{fs}$, the coherence frequency for the $\mathrm{THz}$ waves in the $500 \mu \mathrm{m}$ thick ZnTe crystal of $f_{\text {coh }}=2.8 \mathrm{THz}$ which corresponds to a coherence time of $\tau_{\text {coh }}=210 \mathrm{fs}$, the resolution due to the chirped detection scheme [5] of $\tau_{\mathrm{BW}}=\sqrt{T \tau_{0}}=700 \mathrm{fs}$ with chirped and unchirped length of $T=5.2 \mathrm{ps}$ and $\tau_{0}=130 \mathrm{fs}$, and the spectrometer resolution $\tau_{\lambda}=\Delta \lambda / \eta=190$ fs. This combines to a rms reso-

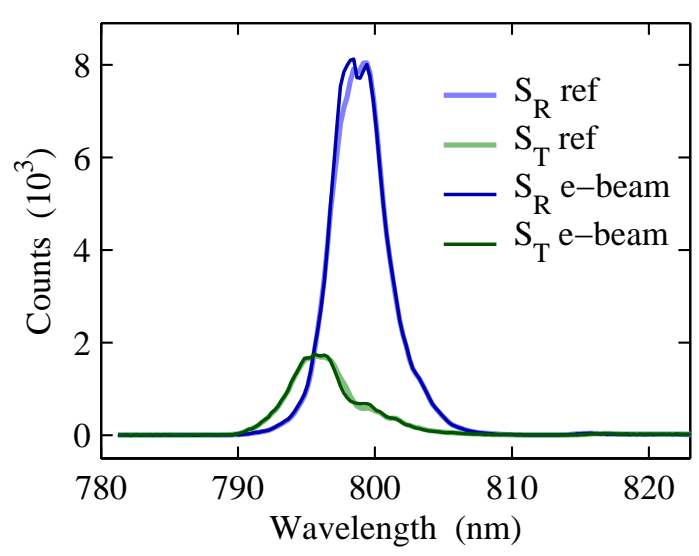

Figure 5: Raw spectra of transmitted (green) and reflected (blue) light. The thick curves are the reference data without beam, the thin curves single-shot spectra with beam. lution of $320 \mathrm{fs}$. When applied to the bunch length from the zero-phasing method, this results in an expected rms length of $725 \mathrm{fs}$ for the electro-optic signal, which is within the fluctuations of the actual measured length. The charge can be calculated to $(180 \pm 35) \mathrm{pC}$ compared to $(290 \pm 15) \mathrm{pC}$ from the Faraday cup.

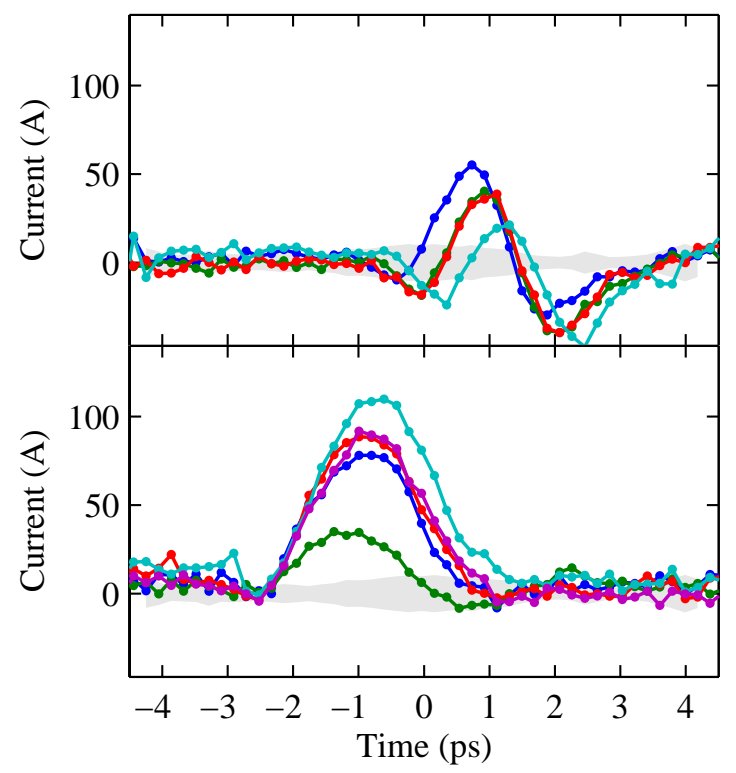

Figure 6: Single-shot electron beam current distribution with 1.67 ps different delay between upper and lower part. The shaded area represents the noise level from spectra without beam.

The single-shot electro-optic method also enables a measurement of the timing jitter between laser and electron beam since the jitter is decoupled from the bunch length. The jitter obtained is $170 \mathrm{fs}$ rms which is of the order of the $200 \mathrm{fs}$ jitter measured between the Ti:Sapphire laser oscillator and the low level RF system.

\section{SUMMARY}

A nonintercepting single-shot electro-optic bunch length measurement has been installed at the DUVFEL accelerator and successfully demonstrated results which agree with theory and with results obtained by zero-phasing.

\section{ACKNOWLEDGEMENTS}

This work was supported by DOE Contract DEAC No. DE-AC02-98CH10886.

\section{REFERENCES}

[1] W. S. Graves et al., PAC 2001, Chicago, June 2001, p. 2224

[2] D.X. Wang et al., Phys. Rev. E 57 (1998) 2283

[3] X. Yan et al., Phys. Rev. Lett. 85 (2000) 3404

[4] I. Wilke et al., Phys. Rev. Lett. 88 (2002) 124801

[5] J. Jiang, X.-C. Zhang, IEEE J. Quant. Electr. 36 (2000) 1214 